\title{
Multiple Signaling Pathways Coordinately Regulate Forgetting of Olfactory Adaptation through Control of Sensory Responses in Caenorhabditis elegans
}

\author{
Tomohiro Kitazono, ${ }^{1}$ Sayuri Hara-Kuge, ${ }^{2,3}$ Osamu Matsuda, ${ }^{2}$ Akitoshi Inoue, ${ }^{1}$ Manabi Fujiwara, ${ }^{1,2}$ and Takeshi Ishihara ${ }^{1,2,3}$ \\ ${ }^{1}$ Graduate School of Systems Life Sciences, ${ }^{2}$ Department of Biology, Faculty of Science, ${ }^{3}$ Core Research for Evolutional Science and Technology, Kyushu \\ University, Fukuoka 8190395, Japan
}

Forgetting memories is important for animals to properly respond to continuously changing environments. To elucidate the mechanisms of forgetting, we used one of the behavioral plasticities of Caenorhabditis elegans hermaphrodite, olfactory adaptation to an attractive odorant, diacetyl, as a simple model of learning. In C. elegans, the TIR-1/JNK-1 pathway accelerates forgetting of olfactory adaptation by facilitating neural secretion from AWC sensory neurons. In this study, to identify the downstream effectors of the TIR-1/JNK-1 pathway, we conducted a genetic screen for suppressors of the gain-of-function mutant of tir-1 (ok1052), which shows excessive forgetting. Our screening showed that three proteins - a membrane protein, MACO-1; a receptor tyrosine kinase, SCD-2; and its putative ligand, HEN-1 - regulated forgetting downstream of the TIR-1/JNK-1 pathway. We further demonstrated that MACO-1 and SCD-2/HEN-1 functioned in parallel genetic pathways, and only MACO-1 regulated forgetting of olfactory adaptation to isoamyl alcohol, which is an attractive odorant sensed by different types of sensory neurons. In olfactory adaptation, odor-evoked $\mathrm{Ca}^{2+}$ responses in olfactory neurons are attenuated by conditioning and recovered thereafter. A $\mathrm{Ca}^{2+}$ imaging study revealed that this attenuation is sustained longer in maco-1 and scd-2 mutant animals than in wild-type animals like the TIR-1/JNK-1 pathway mutants. Furthermore, temporal silencing by histamine-gated chloride channels revealed that the neuronal activity of AWC neurons after conditioning is important for proper forgetting. We propose that distinct signaling pathways, each of which has a specific function, may coordinately and temporally regulate forgetting by controlling sensory responses.

Key words: behavior; Caenorhabditis elegans; forgetting; learning; tyrosine kinase

Significance Statement

Active forgetting is an important process to understand the whole mechanisms of memories. Recent papers have reported that the noncell autonomous regulations are required for proper forgetting in invertebrates. We found that in Caenorhabditis elegans hermaphrodite, the noncell autonomous regulations of forgetting of olfactory adaptation is regulated by three conserved proteins: a membrane protein, MACO-1; a receptor tyrosine kinase, SCD-2: and its ligand, HEN-1. MACO-1 and SCD-2/HEN-1, working in coordination, accelerate forgetting by controlling sensory responses in parallel. Furthermore, temporal regulation of neuronal activity is important for proper forgetting. We suggest that multiple pathways may coordinately and temporally regulate forgetting through control of sensory responses. This study should lead to a better understanding of forgetting in higher organisms.

\section{Introduction}

Active forgetting, in which memories are lost by neuronal regulation, is important for animals to avoid exceeding the storage

Received Jan. 4, 2017; revised Sept. 4, 2017; accepted Sept. 9, 2017.

Author contributions: T.K., A.I., M.F., and T.I. designed research; T.K., S.H.-K., and A.I. performed research; T.K. and 0.M. analyzed data; T.K., M.F., and T.I. wrote the paper.

This work was supported by a Japan Society for the Promotion of Science (JSPS) Grant-in-Aid for JSPS Research Fellow (JP261655 to T.K.); by Grants-in-Aid for Scientific Research (B; 23370002 to T.I.); by Scientific Research on Innovative Areas "Memory Dynamism" (25115009 to T.I.), "Molecular Ethology" (20115003 to T.I.), and "Comprehensive Brain Science Network"; and by Core Research for Evolutional Science and Technology, Japan Science and Technology Agency (JPMJCR12W1 to T.I.). We thank I. Mori for maco-1 strains and plasmids, D. Reiner for scd-2 strains and plasmids, K. Matsumoto and N. Hisamoto for the sek-1 strain, C.I. Bargmann for the HisCl1 plasmid, and A. Miyawaki for YC3.60. We also thank Y. lino for promoters, and N. Sato, N. Yonezawa, and M. Yamaguchi for capacity of the brain and to prevent the interference between old and new memories (Kraemer and Golding, 1997). Although the mechanisms underlying acquisition and consolidation of memories have been extensively studied (Kandel et al., 2014), the

technical assistance. The scd-2 (ok565) strain was provided by the Caenorhabditis Research Center, which is funded by the National Institutes of Health Office of Research Infrastructure Programs (P40 0D010440). We also thank the National Bioresource Project (S. Mitani) for strains.

The authors declare no competing financial interests.

Correspondence should be addressed to Takeshi Ishihara, PhD, Department of Biology, Faculty of Science, Kyushu University, 744 Motooka, Nishi-ku, Fukuoka 8190395, Japan. E-mail: ishihara.takeshi.718@m.kyushu-u.ac.jp. DOI:10.1523/JNEUROSCI.0031-17.2017

Copyright $\odot 2017$ the authors $\quad 0270-6474 / 17 / 3710240-12 \$ 15.00 / 0$ 
mechanisms underlying forgetting are still largely unclear despite its importance.

The molecular mechanisms of active forgetting have been mainly reported in Drosophila. Active forgetting of aversive olfactory memories requires the GTP-binding protein, Rac1, in the mushroom bodies, which are a pair of structures where olfactory learning occurs in the Drosophila brain. In the mushroom bodies, Rac1 regulates forgetting by remodeling the actin cytoskeleton. Rac1 in the mushroom bodies is activated by a small subset of dopaminergic neurons, which reside outside of the mushroom bodies (Shuai et al., 2010; Berry et al., 2012; Cervantes-Sandoval et al., 2016). These studies suggest that active forgetting is regulated in a noncell autonomous manner via neural communication.

One of the behavioral plasticities in C. elegans, olfactory adaptation, has been used as a simple model of learning (Colbert and Bargmann, 1995). Although C. elegans has a simple nervous system, this nematode shows behavioral plasticity to various stimuli. C. elegans can sense many kinds of volatile attractive odorants, which are sensed by two pairs of olfactory sensory neurons, AWA and AWC. These two types of neurons respond to distinct sets of odorants. For example, diacetyl is mainly sensed by AWA neurons, and isoamyl alcohol is sensed by AWC neurons. Furthermore, the regulatory mechanisms of sensation and olfactory adaptation in AWA sensory neurons are different from those in AWC sensory neurons (L'Etoile et al., 2002; Palmitessa et al., 2005; Zhang et al., 2014).

In C. elegans, two regulatory mechanisms have been reported to be implicated in forgetting of olfactory adaptation to the AWAsensed odorant, diacetyl. Hadziselimovic et al. reported that an RNA binding protein, Musashi, regulated forgetting by controlling protein levels of the Arp2/3 actin branching regulator complex in AVA interneurons (Hadziselimovic et al., 2014). We reported that the TIR-1/JNK-1 pathway, composed by an adaptor protein of p38/MAPK pathway, TIR-1 and its downstream proteins, accelerated forgetting by facilitating the secretion of forgetting signals from AWC neurons to AWA neurons (Inoue et al., 2013). Therefore, like in Drosophila, noncell autonomous regulatory mechanisms are also involved in forgetting in C. elegans. However, although dopaminergic signaling is important for forgetting in Drosophila (Berry et al., 2012; Cervantes-Sandoval et al., 2016), the molecular components required for neural communication in C. elegans forgetting is yet unknown.

In this study, we conducted a genetic screen for suppressors of the gain-of-function mutant of tir-1, and found three proteins downstream of the TIR-1/JNK-1 pathway required for forgetting of olfactory adaptation to diacetyl: a membrane protein, MACO-1; a receptor tyrosine kinase, SCD-2; and its putative ligand, HEN-1. Both MACO- 1 and SCD-2 may facilitate forgetting by regulating the odor-evoked $\mathrm{Ca}^{2+}$ response of AWA sensory neurons by parallel pathways. MACO-1 also facilitates forgetting of olfactory adaptation to an AWC-sensed odorant, but SCD-2 and HEN-1 do not. Furthermore, silencing of AWC neurons by histaminegated chloride channels demonstrated that the activity of AWC neurons after conditioning was important for forgetting. In this study, we propose that precise regulation through multiple signaling pathways is used even in forgetting a simple learned behavior, olfactory adaptation.

\section{Materials and Methods}

Strains and culture. The strains used in this study were as follows: wildtype Bristol strain N2, tir-1 (ok1052), tir-1 (tm3036), maco-1 (qj143), maco-1 (nj21), maco-1 (nj34), scd-2 (qj141), scd-2 (sa249), scd-2 (ok565), and hen-1 (tm501). All strains were cultured on nematode growth medium (NGM) plates with Escherichia coli strain OP50 (Brenner, 1974) under standard conditions. All strains were grown at $20^{\circ} \mathrm{C}$. All animals used in this study were hermaphrodite animals.

Behavioral assays. Chemotaxis toward attractive odorants was performed as described previously (Bargmann et al., 1993), although assay plates contained $50 \mathrm{~mm} \mathrm{NaCl}$. The chemotaxis index was calculated as $(A-B) / N$, where $A$ was the number of animals within $1.5 \mathrm{~cm}$ of the odorant spot, $B$ was the number of animals within $1.5 \mathrm{~cm}$ of the control spot, and $N$ was the number of all animals. Before the adaptation and recovery assays, adult worms were washed twice with S-basal buffer (100 mM NaCl, $50 \mathrm{~mm} \mathrm{~K} \mathrm{HPO}_{4}$, $\mathrm{pH}$ 6) containing $0.02 \%$ gelatin, and once with water containing $0.02 \%$ gelatin. In conditioning, animals were pre-exposed to $0.02 \%$ diacetyl or $0.01 \%$ isoamyl alcohol in S-basal buffer with rotation, $4 \mu \mathrm{l}$ of undiluted pyrazine on the lids of $6 \mathrm{~cm}$ unseeded NGM plates, or $2 \mu \mathrm{l}$ of diluted $10 \%$ diacetyl on the lids of $6 \mathrm{~cm}$ OP50seeded NGM plates for $90 \mathrm{~min}$. Next, the worms were washed once with water and allowed to recover on OP50-seeded NGM plates. In histaminegated chloride channel-induced silencing experiments, NGM plates and OP50-seeded NGM plates containing $10 \mathrm{~mm}$ histamine were used. For histamine-mediated silencing during $90 \mathrm{~min}$ of conditioning, worms were transferred to nonhistamine NGM plates from histamine NGM plates after $1 \mathrm{~h}$, to prevent the effect of histamine-induced silencing persisting during recovery. The statistical significance of the results was analyzed using two-way ANOVA followed by post hoc multiple $t$ tests with Bonferroni's correction or Student's $t$ test.

Screening for the suppressor of the gain-of-function mutants of tir-1. The gain-of-function mutant of tir-1 (ok1052) was mutagenized with ethyl methanesulfonate. We collected $7400 \mathrm{~F} 1$ worms and they were allowed to lay F2 worms. In the screen, F2 worms that showed normal chemotaxis to diacetyl were subjected to $1.5 \mathrm{~h}$ of conditioning followed by $4 \mathrm{~h}$ of cultivation with food. After recovery, worms not attracted to diacetyl were allowed to lay F3 worms, and F3 young adults were subjected to the same assays as F2 worms. This protocol was repeated in F3 and F4 generations, and F3 and F4 animals were subjected to the recovery assay twice in $2 \mathrm{~d}$. At the end, worms that showed normal chemotaxis to diacetyl but were not attracted to diacetyl after $4 \mathrm{~h}$ of recovery were chosen as candidates for suppressor mutants of tir-1 (ok1052). After isolating mutant strains, we searched for candidate variants of each strain by whole-genome sequencing and analysis with MAQGene (Mapping and Assembly with Qualities gene) software (Bigelow et al., 2009). We also performed genetic mapping using single nucleotide polymorphisms between N2 and RB1085 (tir-1 (ok1052)), which were identified by whole-genome sequencing, to narrow down the candidate regions for the responsible genes. We confirmed that both qj143 and qj141 mutations reside in the candidate regions.

DNA constructs and germline transformation. To prepare transgenic strains for behavioral assays of rescue experiments and calcium imaging, each DNA fragment was injected at a concentration of $1-5 \mathrm{ng} / \mu \mathrm{l}$ into each mutant animal, respectively. Pmyo-3::gfp, Plin-44::gfp, and Plin-44:: $m$ Cherry were used as injection markers for transgenic strains for behavioral assays and calcium imaging, respectively. Podr-1::maco-1 cDNA was kindly provided by Dr. I. Mori.

Calcium imaging. Calcium imaging of the response to diacetyl in AWA neurons was performed as described previously (Inoue et al., 2013). For conditioning, $0.02 \%$ diacetyl was used, and a $1: 10^{8}$ dilution of the diacetyl solution was used for odor stimulation. $\left(R_{\max }-R_{0}\right) / R_{0}$ was calculated as the peak amplitude of the YFP/CFP ratio $\left(R_{\max }\right)$ during a $10 \mathrm{~s}$ interval after stimulation relative to the mean basal ratio $\left(R_{0}\right)$ during the $10 \mathrm{~s}$ interval preceding the first application of diacetyl. The statistical significance of the results was analyzed using two-way ANOVA followed by post hoc multiple $t$ tests with Bonferroni's correction or Student's $t$ test.

Experimental design and statistical analysis. For all experiments, adult hermaphrodite animals were used. Pmyo-3::gfp, Plin-44::gfp, or Plin-44:: $m$ Cherry were used as injection markers for transgenic strains for behavioral assays and calcium imaging, respectively. All values are presented as mean \pm SEM. Data analysis was performed using BellCurve for Excel (Social Survey Research Information). The statistical significance between the means was compared by two-way ANOVA followed by post hoc multiple $t$ tests with Bonferroni's correction or Student's $t$ test. Exact sample sizes are noted in the figure legends. 


\section{Results}

MACO-1 and SCD-2 suppress the forgetting phenotype of the gain-of-function mutant of tir-1

C. elegans shows strong chemoattraction to the AWA-sensed attractive odorant diacetyl (Fig. 1 A, B, WT Naive). However, after animals were conditioned with diacetyl without food, E. coli, for $1.5 \mathrm{~h}$, they showed much weaker chemoattraction than naive animals (Fig. 1A, $B$, WT Adaptation). This behavioral plasticity is called olfactory adaptation. After animals were cultivated with food without diacetyl for an additional $4 \mathrm{~h}$, animals changed their behavior to show strong chemoattraction again (Fig. $1 A, B$, WT $4 \mathrm{~h}$ recovery). We consider this behavioral change a simple model of forgetting. Our previous study showed that the TIR-1/JNK-1 pathway accelerated forgetting of olfactory adaptation to diacetyl. In loss-of-function mutants of tir-1, olfactory adaptation persists for a longer period than in wild-type animals (Fig. $1 C$, tir-1 (tm3036lf) 4 h recovery). In contrast, tir-1 gain-of-function mutant animals show weak olfactory adaptation. We speculated that this was due to excessive forgetting during conditioning caused by the hyperactivation of TIR-1, although we cannot exclude the possibility that this phenotype may be caused by the defect in establishment of olfactory adaptation (Fig. $1 A, B$, tir-1 (ok1052gf) Adaptation; Inoue et al., 2013).

In this study, to identify downstream effectors of the TIR-1/ JNK-1 pathway, we mutagenized a gain-of-function mutant of tir-1 (ok1052) and screened for individuals exhibiting prolonged retention of diacetyl adaptation following $4 \mathrm{~h}$ of recovery with food. This behavior is similar to that observed in loss-of-function mutants of tir-1, and individuals behaving this way likely bear mutations that suppress TIR-1 activity. In this screen for suppressors, we isolated mutations in maco-1 (qj143) and scd-2 (qj141; see experimental procedures). The mutations in maco- 1 and $s c d-2$ suppressed the weak adaptation phenotype of tir-1 (ok1052g), and they also caused prolonged retention of adaptation to diacetyl after $4 \mathrm{~h}$ recovery with food (Fig. $1 A, B$ ). We also examined the phenotype of single mutants of maco- 1 and $s c d-2$, and these mutants showed a similar phenotype to their double mutants with tir-1 (ok1052gf) after conditioning and $4 \mathrm{~h}$ recovery (Fig. $1 A, B$ ), indicating that the mutations of both maco-1 and $s c d-2$ suppressed the phenotype of tir-1 (ok1052gf) in forgetting. Therefore, in forgetting of olfactory adaptation to diacetyl, both maco-1 and $s c d-2$ mutations are genetically epistatic to a gain-of-function mutation of tir-1.

We also examined the phenotypes of double mutants: a null mutant of maco-1 (qj143) or a null mutant of scd-2 (ok565) (see below) and a loss-of-function mutant of tir-1 or a null mutant of sek-1 $(k m 4)$, which was a downstream effector of TIR-1 in the TIR-1/ JNK-1 pathway (Inoue et al., 2013). In these tests, tir-1 (lf); scd-2 double-mutant animals, sek-1-null mutant animals, and the double mutants of sek-1 (null) and each isolated mutant showed slightly but significantly weaker naive chemoattraction than wild type (Fig. 1D-F, left). Therefore, to equitably compare the phenotypes, we calculated the normalized chemotaxis indexes after conditioning and $4 \mathrm{~h}$ recovery by dividing each chemotaxis index by the naive chemotaxis index (Fig. $1 D-F$, right). maco-1; tir-1 (If) double-mutant animals and maco-1; sek-1 double-null mutant animals showed a similar phenotype as each single mutant animal (Fig. 1C,E, right), suggesting that MACO-1 functions in the same genetic pathway with the TIR-1/JNK-1 pathway. In the scd-2 mutant, although the double mutant with tir-1 (lf) showed significant lower normalized chemotaxis indexes after $4 \mathrm{~h}$ recovery than each single mutant (Fig. $1 D$, right), the double-null mutant with sek-1 showed similar phenotype as the sek-1 mutant
(Fig. $1 F$, right), suggesting that SCD-2 also works in the same genetic pathway with the TIR-1/JNK-1 pathway. Together, these epistasis analyses suggest that MACO- 1 and SCD-2 may function downstream of the TIR-1/JNK-1 pathway. As each single mutant showed defects in forgetting comparable to that observed in the respective double mutants with tir-1 (ok1052gf), we used each single mutant in the following analyses.

Our previous study showed that the prolonged adaptation in tir-1 (lf) mutant animals was suppressed when PKC-1 (gf), which constitutively activates neural secretion (Sieburth et al., 2007), was specifically expressed in AWC neurons (Inoue et al., 2013). These results suggest that the TIR-1/JNK-1 pathway is important for the activation of neural secretion from AWC neurons. To investigate the genetic epistasis between neural secretion from AWC neurons and forgetting effectors MACO-1 and SCD-2, we examined the phenotype of maco-1 and $s c d-2$ mutant animals expressing PKC-1 (gf) in AWC neurons. In maco-1 mutant animals expressing PKC-1 (gf) in AWC neurons, weak chemotaxis after $4 \mathrm{~h}$ recovery was significantly restored, suggesting that MACO-1 functions upstream of neural secretion from AWC neurons (Fig. $1 G$ ). On the other hand, in $s c d-2$ background, the prolonged adaptation was not rescued (Fig. $1 G$ ), suggesting that SCD-2 functions downstream of neural secretion in AWC neurons.

While worms pre-exposed to diacetyl without food showed strong reduction of attraction to diacetyl, animals pre-exposed to diacetyl in the presence of food showed much weaker reduction (Nuttley et al., 2002; Vukojevic et al., 2012). To examine whether the food signals affect olfactory adaptation and its recovery of forgetting defective mutants, we analyzed these behaviors in wild-type, tir-1, maco-1, and scd-2 mutant animals. Wild-type animals conditioned with diacetyl in the presence of food, showed strong chemotaxis like naive animals as reported (Fig. $1 H$ ). Meanwhile, tir-1, maco-1, and scd-2 mutant animals with the same conditioning showed weaker chemotaxis, and these phenotypes were sustained $>4 \mathrm{~h}$ in the presence of food (Fig. $1 H$ ), suggesting that food signals are not sufficient for the suppression of olfactory adaptation in these mutants. In the previous study (Inoue et al., 2013) and in this study (Fig. 1A), we demonstrated that the gain-of-function mutant of tir-1 showed a weak adaptation phenotype, and speculated that hyperactivation of TIR-1 caused excessive forgetting even during conditioning. Together, the chemotactic response after the conditioning may be determined on the balance of forgetting and adaptation during the conditioning. The presence of food during the conditioning may inhibit the olfactory adaptation, but the dysfunction of TIR-1, MACO-1, and SCD-2 prevents this inhibition by the ineffective forgetting during conditioning in the presence of food.

\section{Forgetting of olfactory adaptation to diacetyl is regulated by MACO-1}

MACO-1 in C. elegans is a membrane protein and is reported to be a homolog of macoilin, which is conserved in many species, including humans (Arellano-Carbajal et al., 2011; Miyara et al., 2011). We found that qj143 had a frameshift deletion in the maco-1 gene, which possibly causes a null mutation (Fig. $2 A$ ). To confirm the involvement of MACO-1 in regulation of forgetting, we examined the phenotype of other loss-of-function alleles of maco-1, nj21, and nj34. Both mutant animals exhibited a similar prolonged retention of olfactory adaptation to diacetyl compared with qj143 (Fig. 2B). These results support the hypothesis that MACO-1 regulates forgetting of olfactory adaptation to diacetyl.

To identify the cells in which MACO-1 functions, we performed cell-specific rescue experiments. First, we examined the 
A

B

$\square$ Naïve $\square$ Adaptation $\square$ 4-hour recovery

$\square$ Naiive $\square$ Adaptation $\square$ 4-hour recovery
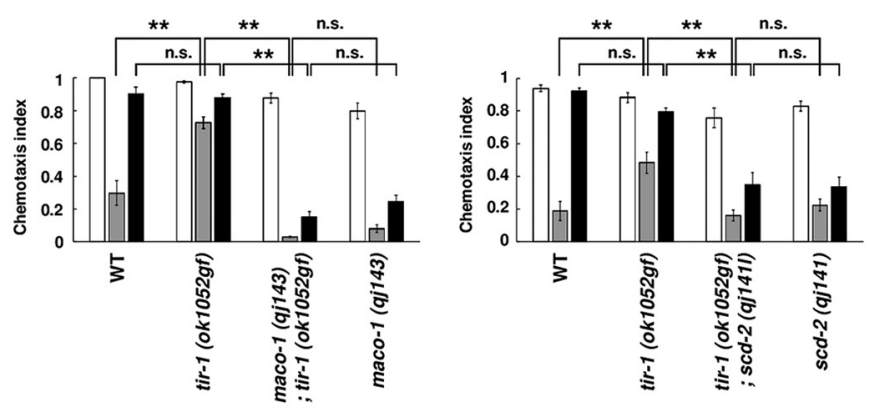

C

D
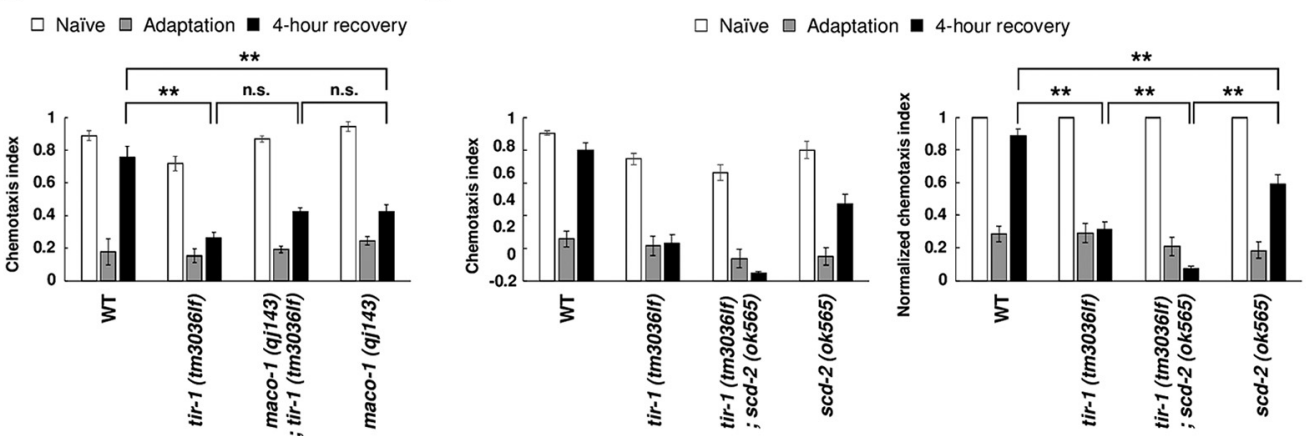

E

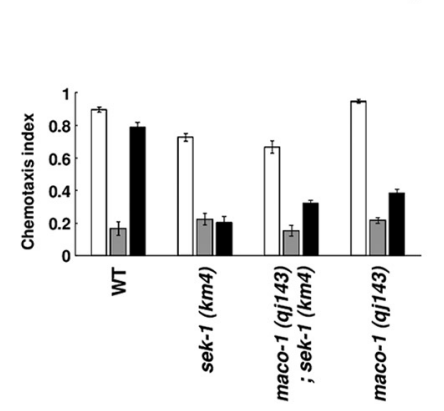

G

$\square$ Naïve $\square$ Adaptation $\square$ 4-hour recovery

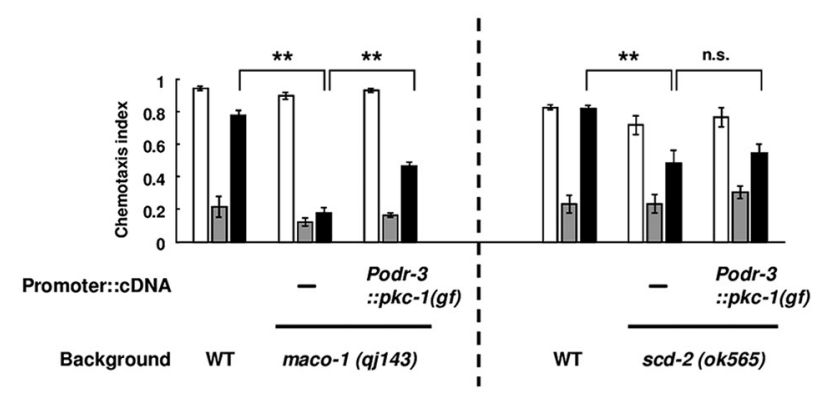

$\mathbf{F}$

$\square$ Naive $\square$ Adaptation $\square$ 4-hour recovery
H

$\square$ Naïve $\square$ Adaptation + Food $\quad$ 4-hour recovery

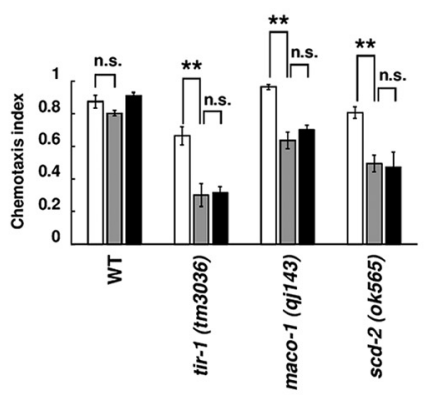

Figure 1. MACO-1 and SCD-2 regulate forgetting of adaptation to diacetyl downstream of TIR-1. A, Genetic interaction between tir-1 (ok 1052gf) and maco-1 (qj143). Chemotaxis of naive, adapted, and 4-h-recovered animals were used ( $n=8,2$-way ANOVA, $\left.F_{\text {strain(3,84) }}=149.8, p<0.001\right)$. B, Genetic interaction between tir-1 (ok1052gf) and scd-2 ( $q j 141 ; n=8,2$-way ANOVA, $\left.F_{\text {strain(3,84) }}=31.4, p<0.001\right)$. C, Genetic interaction between tir- 1 ( $\left.t m 3036 / f\right)$ and maco-1 ( $q j 143 ; n \geq 16,2$-way ANOVA, $\left.F_{\text {strain }(3,276)}=25.9, p<0.001\right)$. D, Genetic interaction between tir-1 (tm3036lf) and scd-2 (ok565). Chemotaxis and normalized chemotaxis were used ( $n \geq 18,2$-way ANOVA, $\left.F_{\text {strain }(3,312)}=28.1, p<0.001\right)$. E, Genetic interaction between sek-1 (km4) and maco-1 (qj743). Chemotaxis and normalized chemotaxis were used ( $n=16,2$-way ANOVA, $\left.F_{\text {strain(3,180) }}=10.7, p<0.001\right)$. $F$, Genetic interaction between sek-1 (km4) and scd-2 (ok565). Chemotaxis and normalized chemotaxis were used ( $n=22$, 2-way ANOVA, $\left.F_{\text {strain }(3,252)}=16.1, p<0.001\right)$. G, Effect of PKC-1 (gf) expression, which activates neural secretion. PKC-1 (gf) was expressed in AWC sensory neurons of maco-1 (qj143) and scd-2 (ok565) mutant animals $\left[n \geq 6\right.$, maco-1 (qj143): 2-way ANOVA, $F_{\text {strain(2,63) }}=48.4, p<0.001 ; s c d-2$ (ok565): $F_{\text {strain(2,45) }}=6, p=0.0049$ ] . $\boldsymbol{H}$, Adaptation to diacetyl and its retention after conditioning with food in wild-type animals, and in tir-1 (tm3036lf), maco-1 (qi143), and scd-2 (ok565) mutant animals ( $n=6$, two-way ANOVA, $\left.F_{\text {treatment }(2,45)}=30.2, p<0.001\right) .{ }^{* *} p<0.01$; post hoc $t$ test with Bonferroni's correction. Error bars represent SEM. 

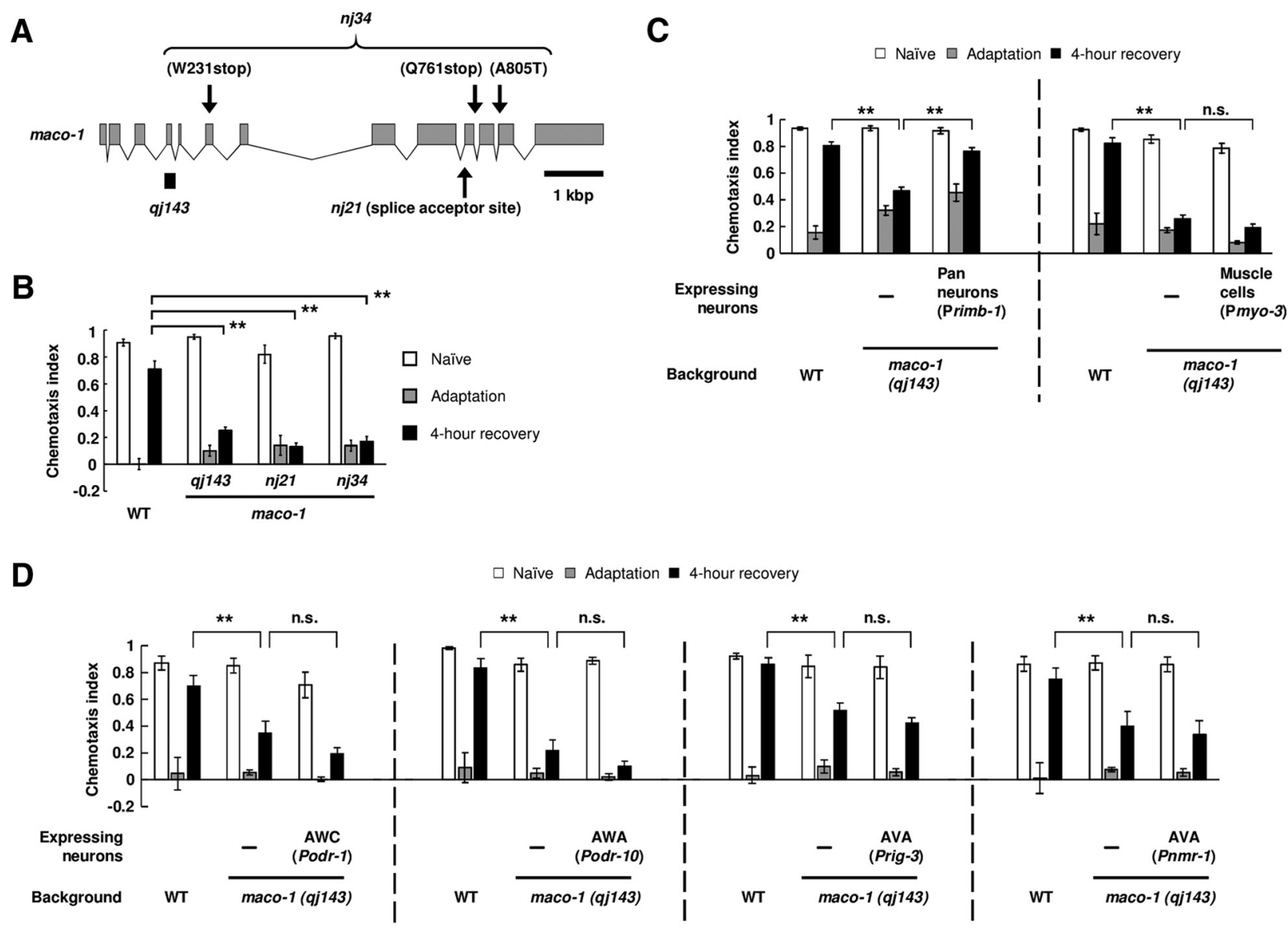

$\mathbf{E}$
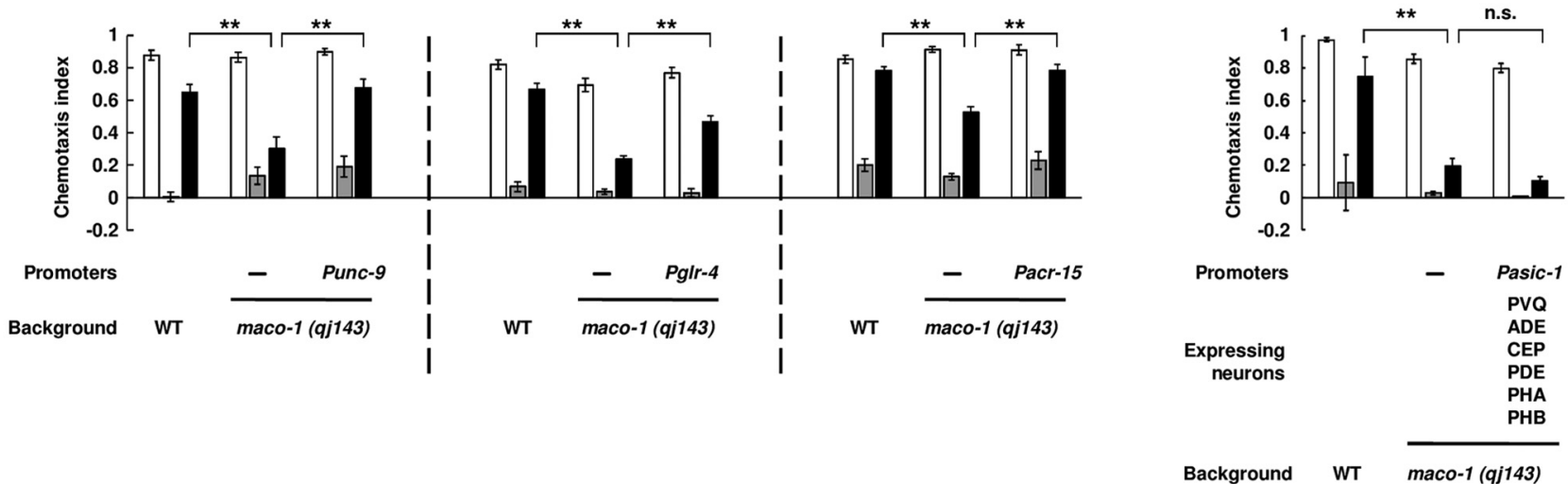

Figure 2. MACO-1 regulates forgetting of adaptation to diacetyl in the nervous system. $A$, Schematic depiction of the maco- 1 gene and of lesions in maco- 1 mutants. Arrows indicate locations of the two maco-1 alleles, and the solid bar indicates a deleted region of qj143. B, Retention of adaptation to diacetyl in maco-1 mutant animals. Chemotaxis of naive, adapted, and 4-h-recovered animals were analyzed $\left(n=6,2\right.$-way ANOVA, $\left.F_{\text {strain }(3,60)}=8.3, p<0.001\right)$. $C-F$, Transgenic rescue of maco-1 (qj143) mutant animals by expressing wild-type gene products under the following promoters: Primb-1, Pmyo-3, Podr-1, Podr-10, Prig-3, Pnmr-1, Punc-9, Pglr-4, Pacr-15, and Pasic-1 (Primb-1: $n=12,2$-way ANOVA, $F_{\text {strain(2.99) }}=11.3, p<0.001$; Pmyo-3: $n \geq 6,2$-way ANOVA, $F_{\text {strain(2,117) }}=316, p<0.001$; Podr-1: $n=8,2$-way ANOVA, $F_{\text {strain }(2,63)}=16.3, p<0.001$; Podr-10: $n=6,2$-way ANOVA, $F_{\text {strain }(2,45)}=36.1, p<0.001 ;$ Prig-3: $n=8,2$-way ANOVA, $F_{\text {strain }(2,63)}=14.2, p<0.001 ;$ Pnmr-1:n $=10,2$-way ANOVA, $F_{\text {strain }(2,81)}=5.2, p<0.001 ;$ Punc- $9: n=8,2$-way ANOVA, $F_{\text {strain }(2,63)}=7.9, p<0.001 ;$ Pglr-4:n=14,2-way ANOVA, $F_{\text {strain }(2,117)}=30.5$, $p<0.001 ;$ Pacr-15: $n=14,2$-way ANOVA, $F_{\text {strain(2,117) }}=10.1, p<0.001$; Pasic-1:n $\geq 4,2$-way ANOVA, $\left.F_{\text {strain(2,75) }}=28.2, p<0.001\right) .{ }^{* *} p<0.01$; posthoct test with Bonferroni's correction $(\boldsymbol{B}-\boldsymbol{F})$. Error bars represent SEM.

behavioral phenotype of maco-1 mutant animals expressing wildtype MACO-1 in the nervous system and muscle cells, and found that expression in the nervous system significantly rescued the prolonged retention phenotype, but expression in muscle cells did not (Fig. 2C). These results suggest that MACO-1 regulates forgetting in the nervous system. Next, we examined whether MACO-1 functioned in AWA, AWC, or AVA neurons individually, which were each reported to regulate forgetting of olfactory adaptation to diacetyl (Inoue et al., 2013; Hadziselimovic et al., 2014). However, the expression of wild-type MACO-1 in each of 
Table 1. The expression in the nervous systems of Punc-9, PgIr-4, and Pacr-15

Punc-9 Phsh, ADA, ADE, ADL, AIN, AIY, ALM, AUA, AVA, AVD, AVH, AVJ, AVK, AVM, AWB, BDU, CAN, CEP, DAn, DBn, DDn, DVB, DVC, FLP, HSN, IL1, IL2, LUA, OLL, PDA, PDB, PDE, PHA, PHB, PHC, PLM, PLN, PVC, PVD, PVM, PVN, PVP, PVQ, PVR, PVT, PVW, RIB, RIC, RIF, RIP, RIS, RME, SDQ, URA, URB, VAn, VBn, VCn, VDn, $M 5,11,16$, NSM

Pglr-4 AVA, RMD, SMD, SAA, SIB, RIB, RIM, AVH, FLP, RMG, DVA, AUA, PVD, URY, URA, $S A B, R I F, D B, P V U$

Pacr-15 AVA, AVB, DVA, I5, RID, RIM, PVQ, SAA, SIA, SIB, SMD

The expression patterns of three promoters have been reported in Altun et al. (2009) for Punc-9, Brockie et al. (2001) for Pglr-4, and Feng et al. (2006) for Pacr-15.

the neurons did not rescue the mutant phenotype (Fig. 2D), suggesting that expression of MACO-1 in each neuron is insufficient for regulation of forgetting. By additional rescue experiments, we further examined which neurons are involved in forgetting. Among various promoters, we found that the expression from the unc-9, glr-4, or acr-15 promoters could rescue the mutant phenotype (Fig. 2E). Although all of these three promoters give expression in AVA neurons (Table 1; Brockie et al., 2001; Feng et al., 2006; Altun et al., 2009), the expression of MACO-1 in AVA neurons could not rescue the mutant phenotype as described above (Fig. 2D). We also examined whether the mutant phenotype was rescued by the expression of MACO-1 in PVQ neurons, in which $u n c-9$ and acr-15 promoters give expression among three promoters shown above (Table 1), but the expression in these neurons could not restore the phenotype (Fig. 2E). These results imply that MACO-1 may function in a subset of neurons for regulation of forgetting.

\section{Forgetting of olfactory adaptation to diacetyl is also regulated by the SCD-2/HEN-1 pathway}

SCD-2 is a homolog of the anaplastic lymphoma kinase (ALK) receptor tyrosine kinase in C. elegans, and is conserved across species including humans (Reiner et al., 2008). We found that qj141 had a point mutation in the kinase domain of the scd-2 gene (Fig. 3A). To confirm involvement of SCD-2 in regulation of forgetting, we examined the phenotype of a loss-of-function mutant allele, sa249, and a null mutant allele, ok565. Mutant animals with these alleles exhibited prolonged retention of olfactory adaptation to diacetyl similar to qj141 (Fig. 3B). These results support the hypothesis that SCD-2 regulates forgetting of olfactory adaptation to diacetyl. We also found that the phenotype of $s c d-2$ mutants was significantly rescued by pan-neuronal expression of the wild-type SCD-2 (Fig. 3C). These results suggest that SCD-2 functions in the nervous system in forgetting.

In response to harsh environments, C. elegans larvae enter into the developmentally arrested diapause dauer state. SCD-2 was first identified as a regulator of dauer formation (Reiner et al., 2008). Furthermore, we have previously shown that SCD-2 in AIA interneurons modulates the integration of two conflicting sensory cues: an attractive odorant, diacetyl, and an aversive stimulus, copper ion (Shinkai et al., 2011). While regulating this response, SCD-2 functions in conjunction with its putative ligand HEN-1. HEN-1 has a homology to Jeb, which is a ligand for a Drosophila homolog of ALK (Krieger and Herz, 1994; Weiss et al., 2001; Ishihara et al., 2002). Therefore, we examined whether HEN-1 also regulated forgetting of olfactory adaptation to diacetyl. hen-1 (tm501)-null mutant animals also showed the same prolonged retention phenotype as $s c d-2$ mutant animals, and this phenotype was rescued by pan-neuronal expression of wild-type HEN-1 protein (Fig. 3D). In dauer formation and sensory integration, SCD-2 and HEN-1 function in the same genetic pathway. Thus, to test whether these two proteins function in the same genetic pathway in forgetting olfactory adaptation, we examined the phenotype of the scd-2; hen-1 double mutant. The double-mutant animals showed a similar phenotype as $s c d-2$ mutant animals, suggesting that SCD-2 and HEN-1 function in the same genetic pathway (Fig. $3 E$ ). These results suggest that, in the nervous system, SCD-2 and HEN-1 regulate forgetting by the same genetic pathway.

\section{MACO-1 and SCD-2 regulate forgetting in distinct genetic pathways}

The behavioral assays of the double mutants with tir- 1 ( $g f$ ) showed that maco- 1 and $s c d-2$ mutations were epistatic to a gain-of-function mutation of tir-1. To further investigate the function of MACO-1 and SCD-2, we conducted two behavioral analyses. First, we examined whether MACO-1 and SCD-2 functioned in the same genetic pathway or in distinct genetic pathways. To test this, we analyzed the phenotype of maco-1; scd-2 double-null mutant animals. maco-1; scd-2 double-null mutant animals showed slightly but significantly weaker chemoattraction than wild type and each single mutant (Fig. $4 A$, left), and thus, we compared the normalized chemotaxis indexes described above. After $4 \mathrm{~h}$ recovery with food after conditioning, maco-1; scd-2 double-mutant animals showed significant lower normalized chemotaxis indexes than each single mutant (Fig. 4A, right), indicating that this double mutant was more weakly recovered from olfactory adaptation than each single mutant. This result suggests that the maco-1; scd-2 double mutant exhibits an additive phenotype, and thus, MACO-1 and SCD-2 may regulate forgetting of olfactory adaptation by distinct genetic pathways. Considering that, together with other epistasis analyses (Fig. $1 A-G$ ), forgetting of olfactory adaptation may be regulated by complicated signaling pathway downstream of the TIR-1/JNK-1 pathway, which is discussed below.

Next, to determine whether MACO-1 and SCD-2 generally regulate forgetting of olfactory adaptation, we examined whether mutant animals of maco- 1 and $s c d-2$ showed prolonged retention of olfactory adaptation to the AWC-sensed attractive odorant isoamyl alcohol. The TIR-1/JNK-1 pathway regulates forgetting of olfactory adaptation in AWC neurons as well as in AWA neurons (Inoue et al., 2013). The regulatory mechanisms of sensation and olfactory adaptation are different between these two types of neurons (L'Etoile et al., 2002; Palmitessa et al., 2005; Zhang et al., 2014). Our behavioral analyses showed that maco-1 mutant animals showed prolonged adaptation to isoamyl alcohol (Fig. 4B), suggesting that MACO-1, like the TIR-1/JNK-1 pathway, facilitates forgetting of olfactory adaptation to both odorants. In contrast, $s c d-2$ mutant animals showed similar recovery as wild-type animals (Fig. 4B), suggesting that SCD-2 facilitates only forgetting of olfactory adaptation to diacetyl. In addition, hen-1 mutant animals showed similar phenotype in the same manner as scd-2 mutant animals (Fig. 4B), suggesting that HEN-1 functions in the same genetic pathway in forgetting of olfactory adaptation in AWC neurons as well. Together, two types of signaling pathways may exist downstream of the TIR-1/JNK-1 pathway. One pathway may regulate forgetting of olfactory adaptation in both AWA and AWC neurons, and the other may regulate forgetting of adaptation only in AWA neurons.

\section{MACO-1 and SCD-2 regulate the sensory $\mathrm{Ca}^{2+}$ response}

Our previous study demonstrated that the change in behavioral response to diacetyl after conditioning and recovery was correlated to the alteration of diacetyl-evoked $\mathrm{Ca}^{2+}$ response in AWA neurons, and that prolonged adaptation in the TIR-1/JNK-1 pathway mutants is due to the slower recovery of the sensory response to diacetyl after conditioning (Inoue et al., 2013). To examine whether 


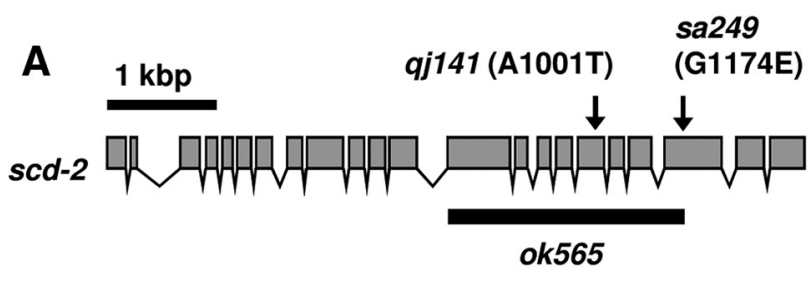

B
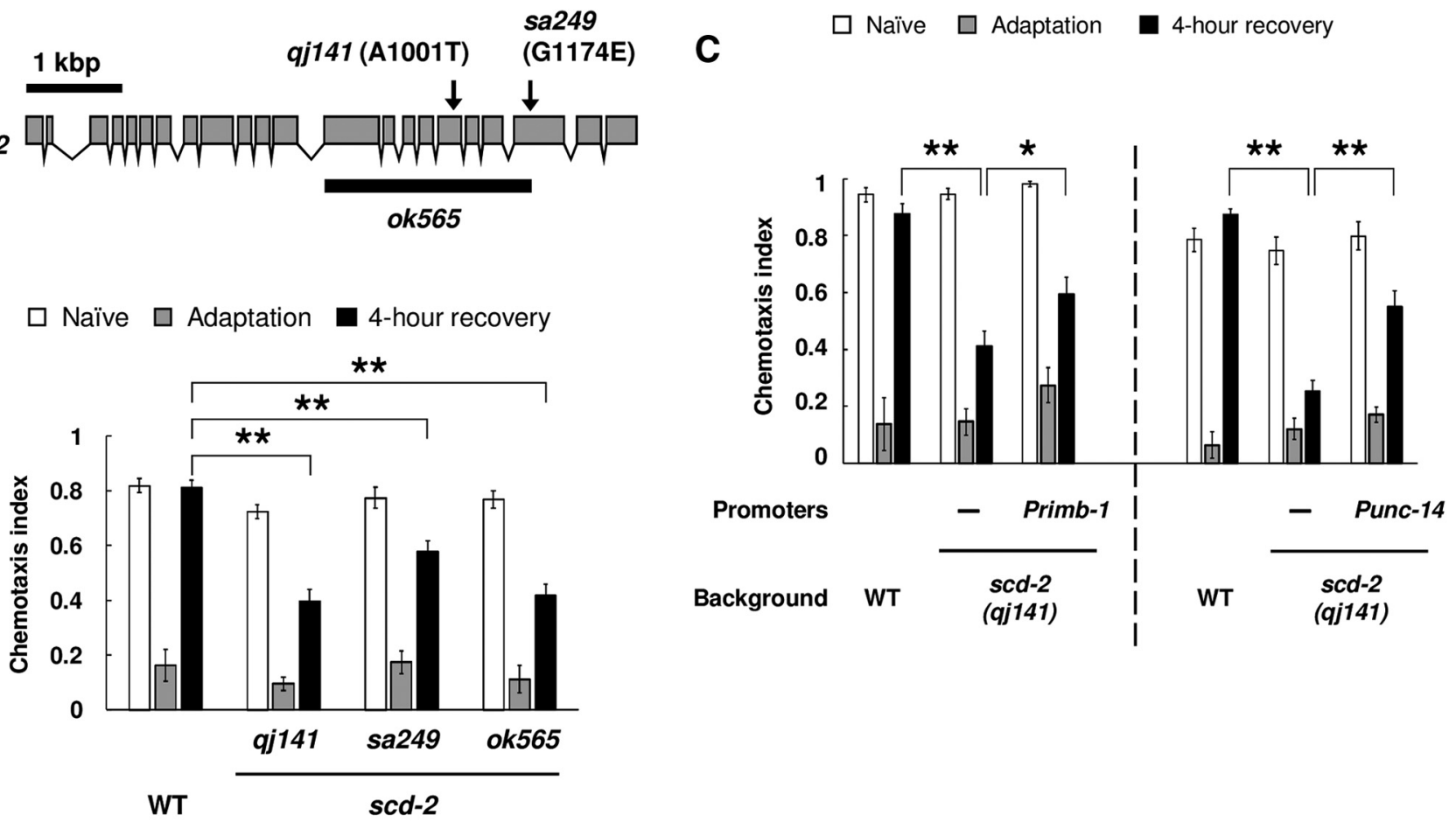

D

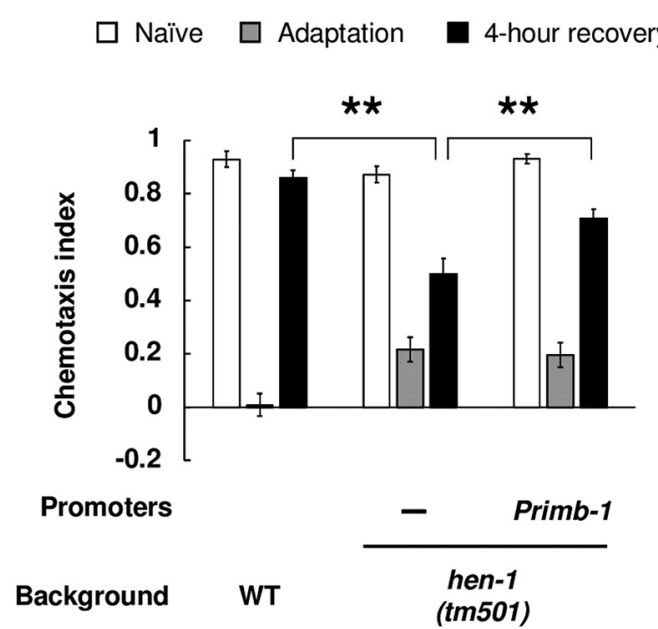

E

Naîve $\square$ Adaptation $\square$ 4-hour recovery

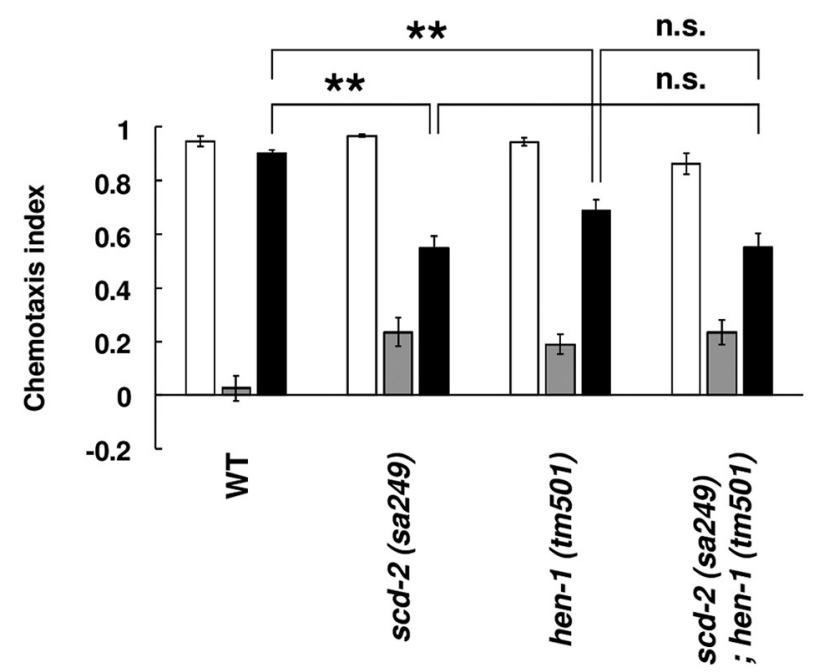

Figure 3. SCD-2 and HEN-1 regulate forgetting of adaptation to diacetyl in the same signaling pathway. $A$, Schematic depiction of the scd-2 gene and of lesions in scd- 2 mutants. Arrows indicate the sites of the two scd-2 alleles, and the solid bar indicates a deleted region of $o k 565$. B. Retention of adaptation to diacetyl in scd-2 mutant animals. Chemotaxis of naive, adapted, and 4-h-recovered animals were analyzed $\left(n=14,2\right.$-way ANOVA, $\left.F_{\text {strain(3,156) }}=15.3, p<0.001\right)$. C, Transgenic rescue of $s c d-2$ ( $\left.q j 141\right)$ mutant animals by expressing wild-type gene products under the following promoters: Primb-1 and Punc-14 (Primb-1: $n=12,2$-way ANOVA, $F_{\text {strain(2,99) }}=11.7, p<0.001$; Punc-14: $n=6,2$-way ANOVA, $F_{\text {strain }(2,45)}=17.8, p<0.001$ ). D, Retention of adaptation to diacetyl in hen-1 (tm501) mutant animals $(n=8)$, and transgenic rescue of hen-1 (tm501) mutant animals by expressing wild-type gene products under a pan-neuronal promoter $\left(n=10,2\right.$-way ANOVA, $\left.F_{\text {strain(2.81) }}=3.8, p=0.0259\right)$. $\boldsymbol{E}$, Retention of adaptation to diacetyl in wild-type, scd-2 (sa249); hen-1 (tm501), and each single mutant ( $n=12$, 2-way ANOVA, $\left.F_{\text {strain(3,132) }}=7.6, p<0.001\right) .{ }^{*} p<0.05,{ }^{* *} p<0.01$; post hoc $t$ test with Bonferroni's correction $(\boldsymbol{B}-\boldsymbol{E})$. Error bars represent SEM.

MACO-1 and SCD-2 also regulate changes in the sensory response, we observed the diacetyl-evoked $\mathrm{Ca}^{2+}$ response in AWA neurons using a $\mathrm{Ca}^{2+}$ indicator, YC3.60 (Nagai et al., 2004). Naive wild-type animals showed an increase in $\mathrm{Ca}^{2+}$ in response to diacetyl stimulus. This response was weakened after conditioning, and recovered after $4 \mathrm{~h}$ cultivation with food (Fig. $5 A, B$ ). In contrast, both maco-1 and scd-2 mutants exhibited a similar weakened $\mathrm{Ca}^{2+}$ response after conditioning as wild type, but these weakened responses were not recovered after $4 \mathrm{~h}$ cultivation with food (Fig. $5 A, B$ ). These results suggest that MACO-1 and SCD-2 also regulate forgetting of olfactory adaptation to diacetyl by controlling the sensory response to diacetyl in AWA neurons.

Next, we analyzed whether the expression of a wild-type gene can rescue the sensory response after the recovery in maco- 1 and $s c d-2$ mutants. In our previous study, the expression of wild-type TIR-1 in AWC neurons rescued the slower recovery of $\mathrm{Ca}^{2+}$ 
A

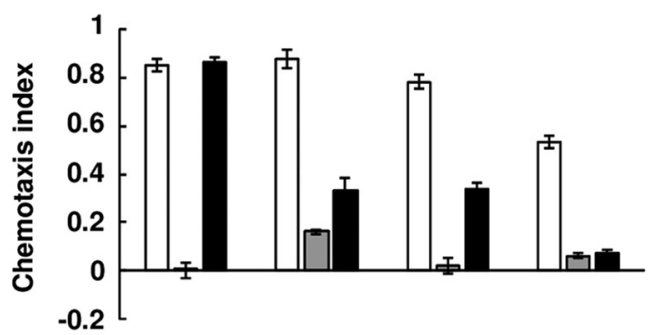

5
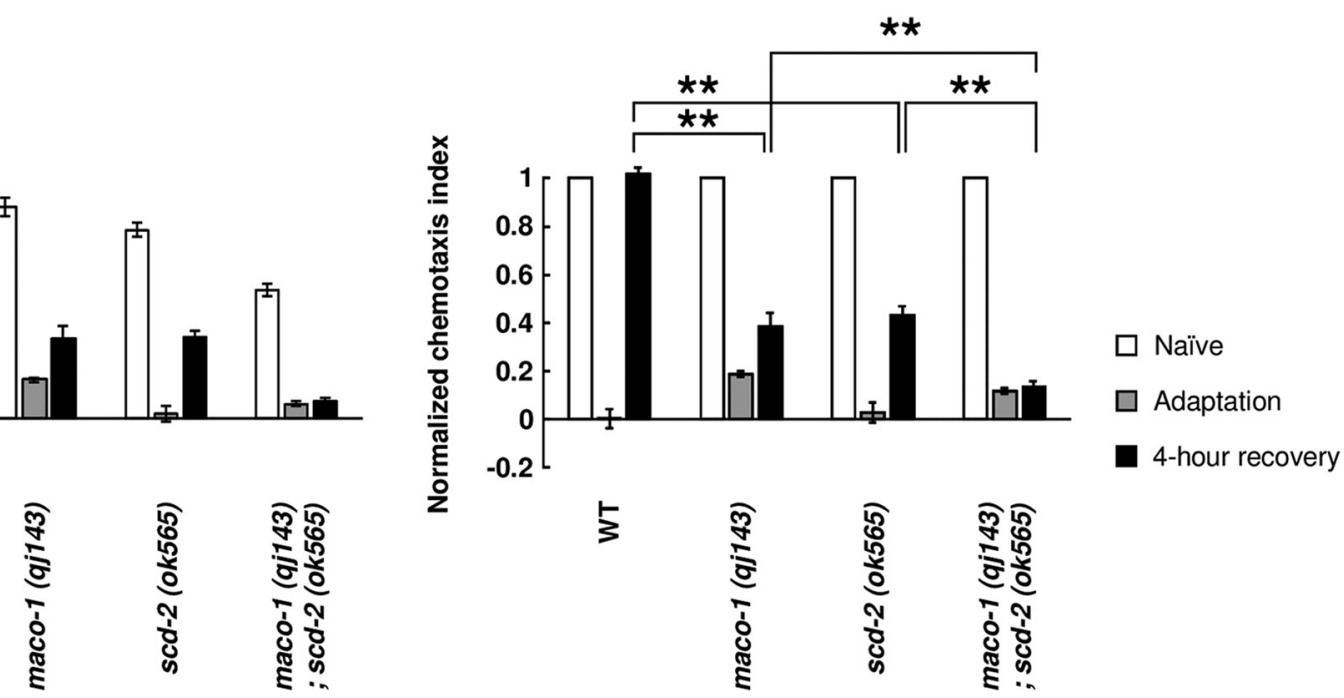

B

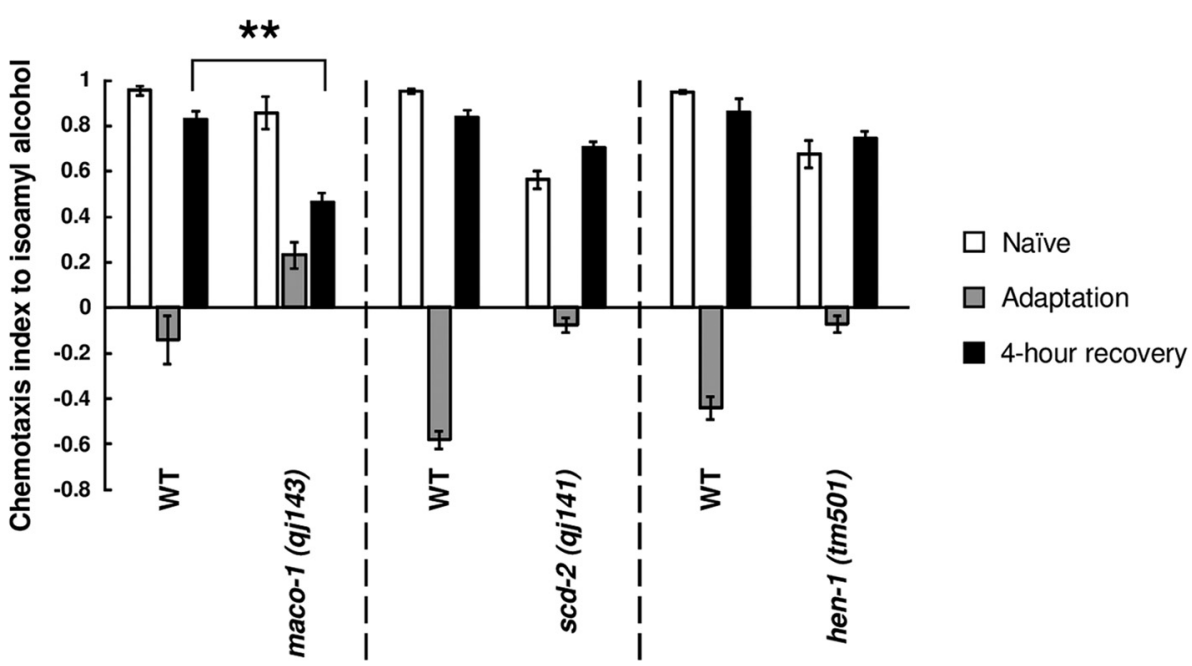

Figure 4. MACO-1 and SCD-2/HEN-1 function in the distinct signaling pathway. A, Retention of adaptation to diacetyl in wild-type and in maco-1 ( $q j 143)$; scd-2 (ok565), and each single mutant $\left(n \geq 8,2\right.$-way ANOVA, $\left.F_{\text {strain(2,132) }}=457.2, p=0.0049\right) . B$, Retention of adaptation to isoamyl alcohol in wild-type, maco-1 (qj143), scd-2 (qj141), and hen-1 (tm501) animals $(n \geq 6)$. ${ }^{* *} p<0.01$; post hoc $t$ test with Bonferroni's correction $(\boldsymbol{A})$ or Student's t test $(\boldsymbol{B})$. Error bars represent SEM.

response in AWA neurons as behavior (Inoue et al., 2013). In the maco-1 mutant, we examined whether pan-neuronal, AWAspecific, or AWC-specific expression of wild-type MACO-1 rescued the $\mathrm{Ca}^{2+}$ response in AWA neurons, and found that none of them rescued the mutant phenotype of AWA sensation (Fig. $5 C$ ), although the pan-neuronal expression of MACO-1 rescued the behavioral phenotype of maco-1. This suggests that the recovery of the sensory response might not be required for the behavioral recovery (see Discussion), although the expression of MACO-1 in the nervous system could be inadequate. Meanwhile, in $s c d-2$ mutants, we found that the slower recovery of $\mathrm{Ca}^{2+}$ response in AWA neurons was rescued by pan-neuronal expression and AWA-specific expression of SCD-2 (Fig. 5D), but not by AWCspecific expression, suggesting that $\mathrm{SCD}-2$ regulates the sensory responses after the recovery in AWA neurons.

\section{AWC neurons accelerate forgetting of olfactory adaptation during recovery}

Our previous study demonstrated that the TIR-1/JNK-1 pathway in AWC neurons controlled forgetting of olfactory adaptation in the mature nervous system (Inoue et al., 2013). However, it is still unclear exactly when activation of the forgetting process in AWC neurons functions in the learning and forgetting process. To elucidate this, we introduced the Drosophila histamine-gated chloride channel to AWC neurons, and specifically silenced these neurons by application of exogenous histamine during conditioning or recovery (Pokala et al., 2014). Because AWC malfunction causes a mild chemotaxis defect to diacetyl (Chou et al., 2001), we used pyrazine, which is also sensed by AWA neurons, to evaluate the effect of silencing by histamine-gated chloride channel. tir-1 mutant animals showed prolonged retention of adaptation to pyrazine as diacetyl (Fig. 6A). First, we observed that the application of histamine to these transgenic animals during both conditioning and recovery caused prolonged retention of olfactory adaptation (Fig. 6B), suggesting that, consistent with our previous results, inactivation of AWC neurons causes prolonged retention of adaptation. Next, we applied histamine to the transgenic animals during either conditioning or recovery. Application of histamine during recovery, but not during conditioning, caused prolonged retention of adaptation (Fig. 6B). Furthermore, when AWC 
A

Naïve
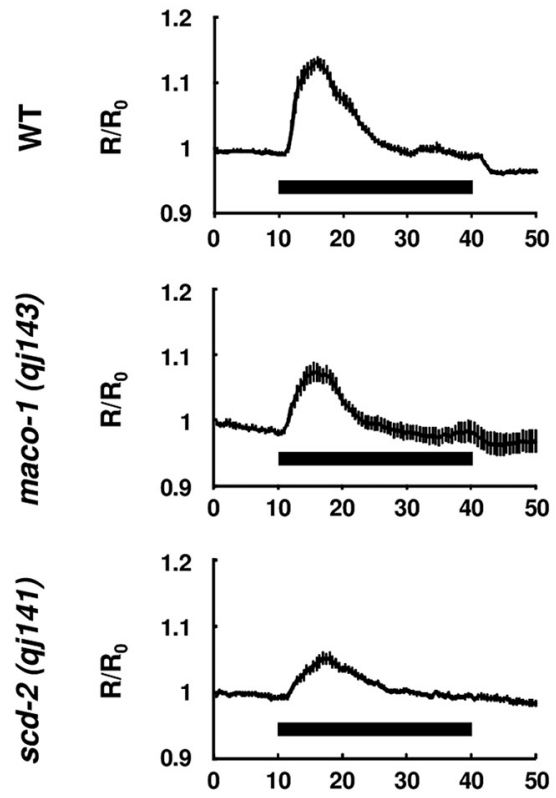

B

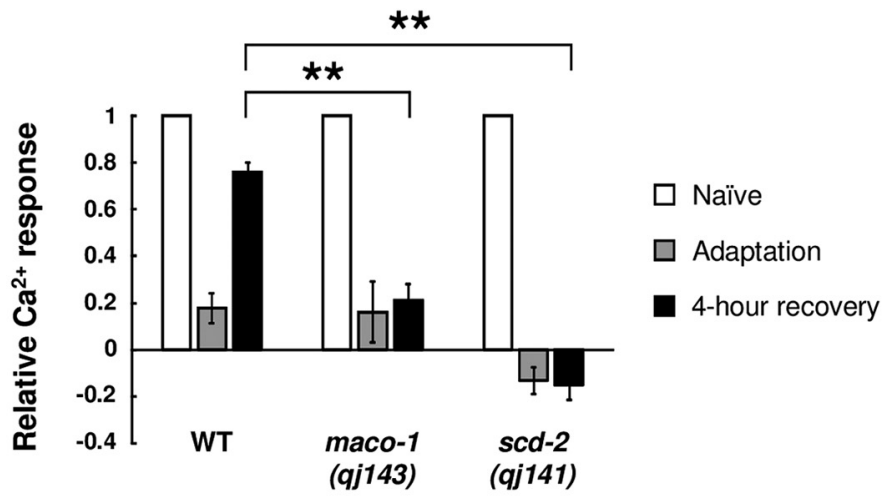

C

Naïve $\square$ Adaptation $\square$ 4-hour recovery

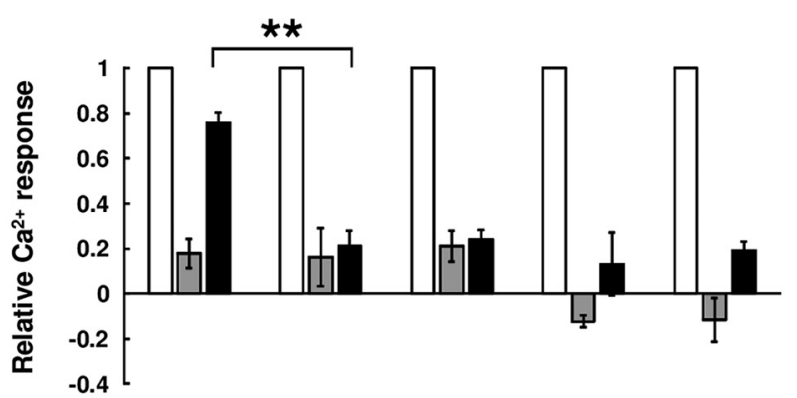

Expressing

neurons 4-hour recovery
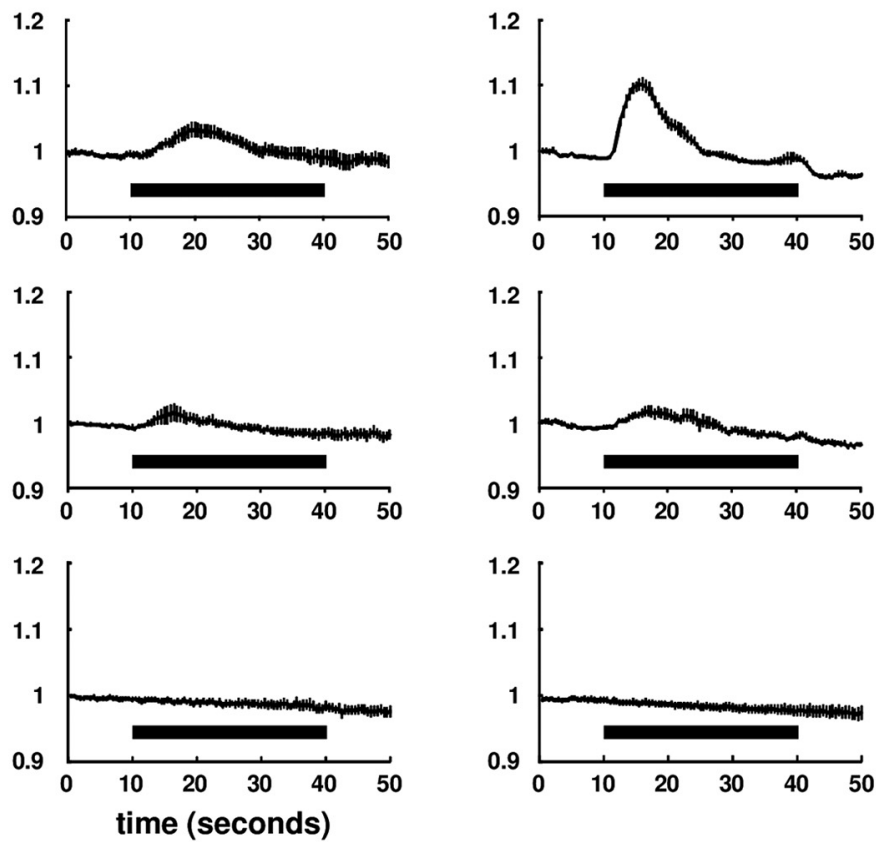

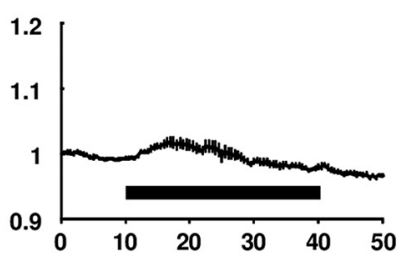

D

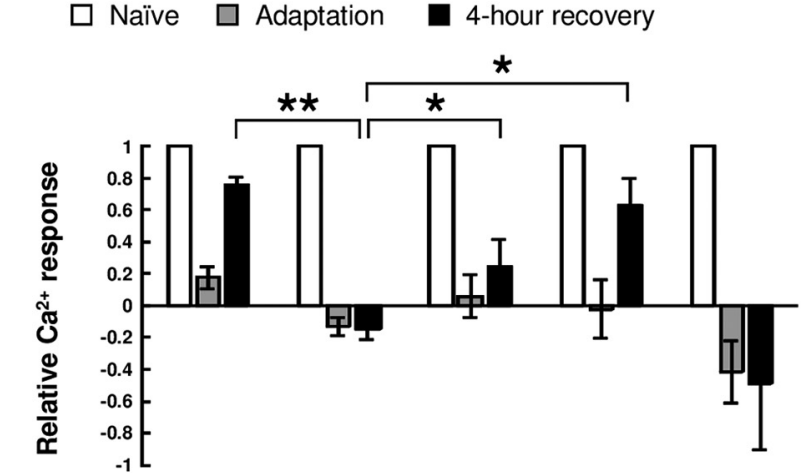

Expressing

neurons

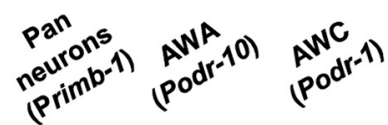

scd-2 (qj141)

Background

Figure 5. $\mathrm{Ca}^{2+}$ responses of AWA sensory neurons after adaptation and recovery. $\boldsymbol{A}$, Averaged traces of $\mathrm{Ca}^{2+}$ response in AWAs in naive, diacetyl-adapted, and 4-h-recovered animals ( $n \geq 8$ ). Timing of the diacetyl stimulation is shown as black bars. $R$ represents the YFP/CFP ratio, and $R_{0}$ represents the averaged ratio 30 s before diacetyl stimulation. $B$, Quantification of $C{ }^{2+}$ responses of wild-type, maco-1 (qj143), and scd-2 (qj141) animals ( $n \geq 8,2$-way ANOVA, $\left.F_{\text {strain(2,114) }}=88.7, p<0.001\right)$. Data were normalized to the averages in the naive animals. $C$, Transgenic rescue of maco-1 (qj143) mutant animals by expressing wild-type gene under a pan-neuronal promoter ( $n \geq 5,2$-way AN0VA, $\left.F_{\text {strain }(4,148)}=88.2, p<0.001\right)$. $D$, Transgenic rescue of scd-2 (qj141) mutant animals by expressing wild-type gene under rimb- 1 and odr-10 promoters $\left(n \geq 6,2\right.$-way ANOVA, $\left.F_{\text {strain }(4,150)}=7.4, p<0.001\right)$. ${ }^{*} p<0.05,{ }^{* *} p<0.01 ; p 0$ st hoc $t$ test with Bonferroni's correction $(B-D)$. Error bars represent SEM. 
A

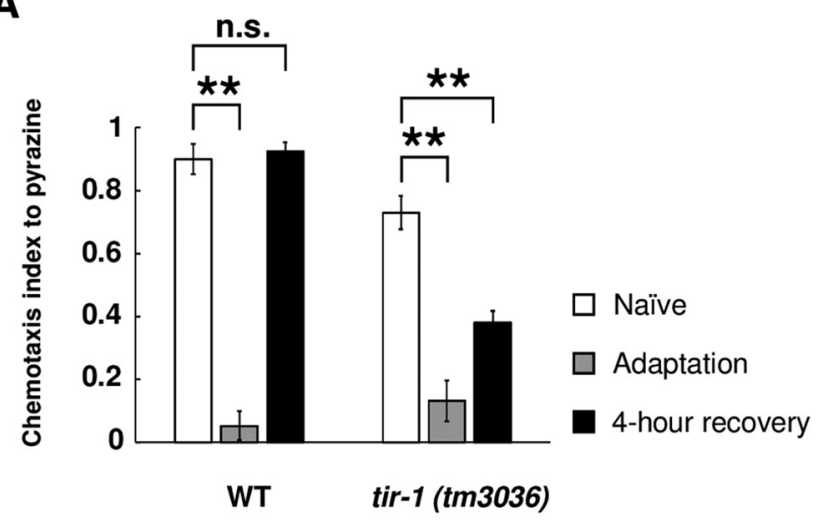

C

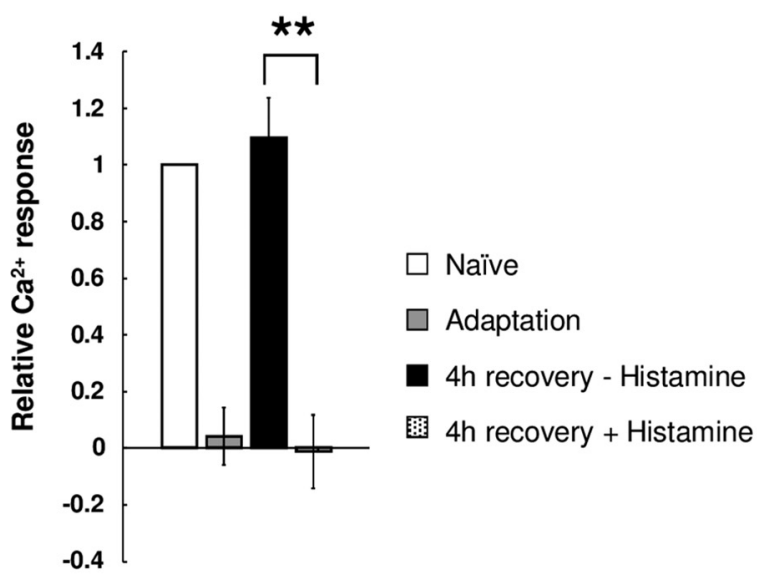

B

Naïve $\square$ Adaptation $\square$ 4-hour recovery

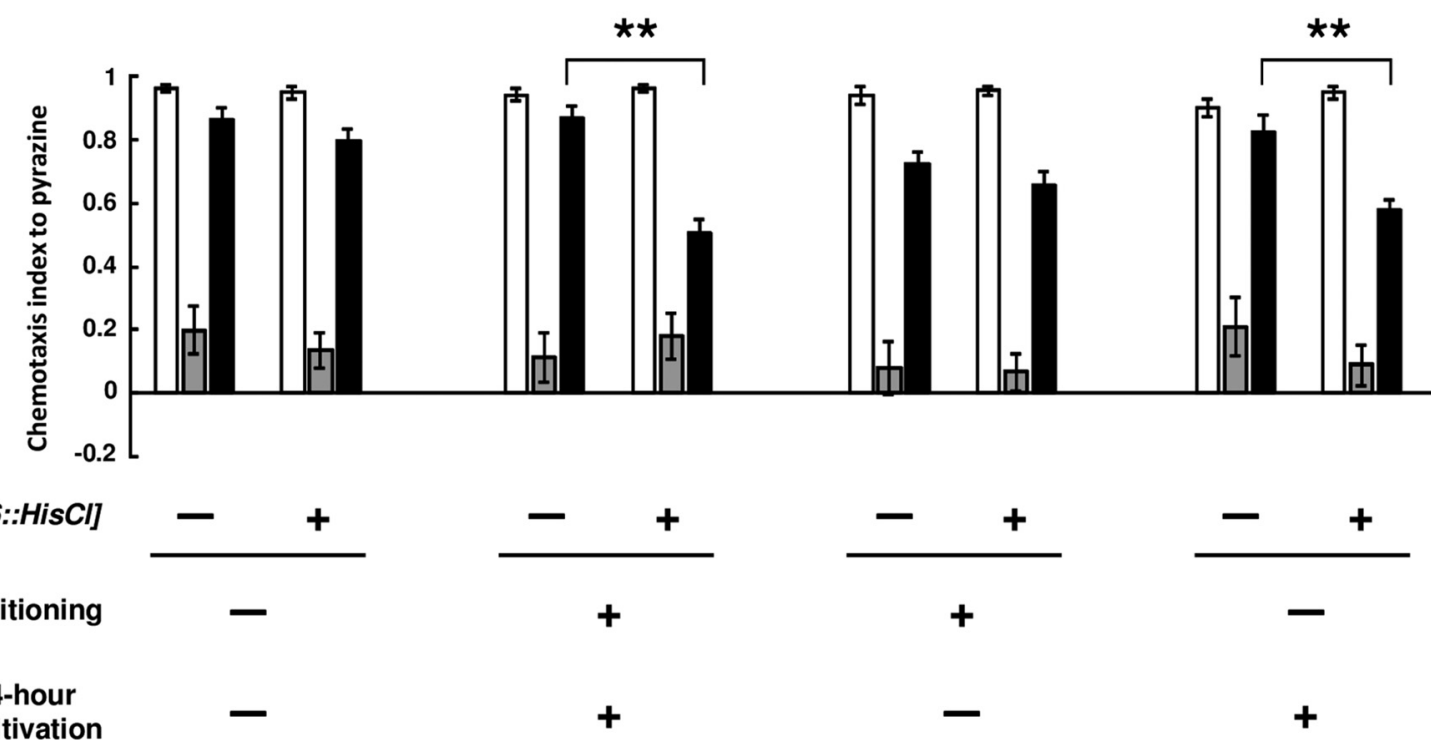

Figure 6. The activity of AWC neurons during recovery is important for forgetting adaptation. $A$, Retention of olfactory adaptation to pyrazine in wild-type and $t$ ir- $7(t m 3036)$ animals $(n=6)$. $B$, Retention of olfactory adaptation to pyrazine in wild-type animals expressing the Drosophila histamine-gated chloride channel in AWC neurons. The timing of application of histamine is shown in the figure. Chemotaxis of naive, adapted, and 4-h-recovered animals was analyzed $(n=12)$. C, Quantification of $\mathrm{Ca}^{2+}$ responses in AWA neurons of wild-type animals expressing histaminegated chloride channels in AWC neurons $(n \geq 8)$. Data were normalized to the averages in the naive animals. ${ }^{* *} p<0.01 ; t$ test with Bonferroni's correction $(\boldsymbol{A})$ or Student's $t$ test $(\boldsymbol{B}, \boldsymbol{C})$. Error bars represent SEM.

neurons were silenced by histamine after diacetyl conditioning, we could not observe the diacetyl-evoked $\mathrm{Ca}^{2+}$ response in AWA neurons after $4 \mathrm{~h}$ recovery (Fig. $6 \mathrm{C}$ ). These results suggest that the neuronal activities of AWC neurons during recovery is important for forgetting of olfactory adaptation.

\section{Discussion}

Active forgetting is important for animals to survive in continuously changing environments, but the regulatory mechanisms guiding the process are largely unknown. In this study, we demonstrated that MACO- 1 and SCD-2/HEN-1 regulated forgetting of olfactory adaptation in distinct genetic pathways. These proteins accelerate forgetting through parallel control of the sensory response of the olfactory neurons. This study also suggests that the activity of AWC neurons after conditioning is important for forgetting.
MACO-1 may regulate the release of forgetting signals from AWC neurons and might compensate downstream of AWA sensation

Our epistasis analyses and cell-specific rescue experiments suggested that MACO-1 has at least two functions in forgetting. First, MACO-1 may facilitate the release of forgetting signals from AWC neurons, which the TIR-1/JNK-1 pathway may regulate (Fig. 7). Second, in behavior, pan-neuronal expression of MACO-1 rescued prolonged retention of adaptation after $4 \mathrm{~h}$ cultivation with food, even though it did not rescue weakened $\mathrm{Ca}^{2+}$ response in AWA neurons. This raises a possibility that not only the synaptic release from AWC neurons, but also downstream mechanisms of AWA sensation are directly or indirectly regulated by MACO-1 and thereby the lack of AWA sensory response might be compensated by the functions of MACO-1 (Fig. 7). This hypothesis is consistent with that MACO-1 functions in subsets of neurons for the regulation of forgetting, although, the expression of $\mathrm{Ca}^{2+}$ 


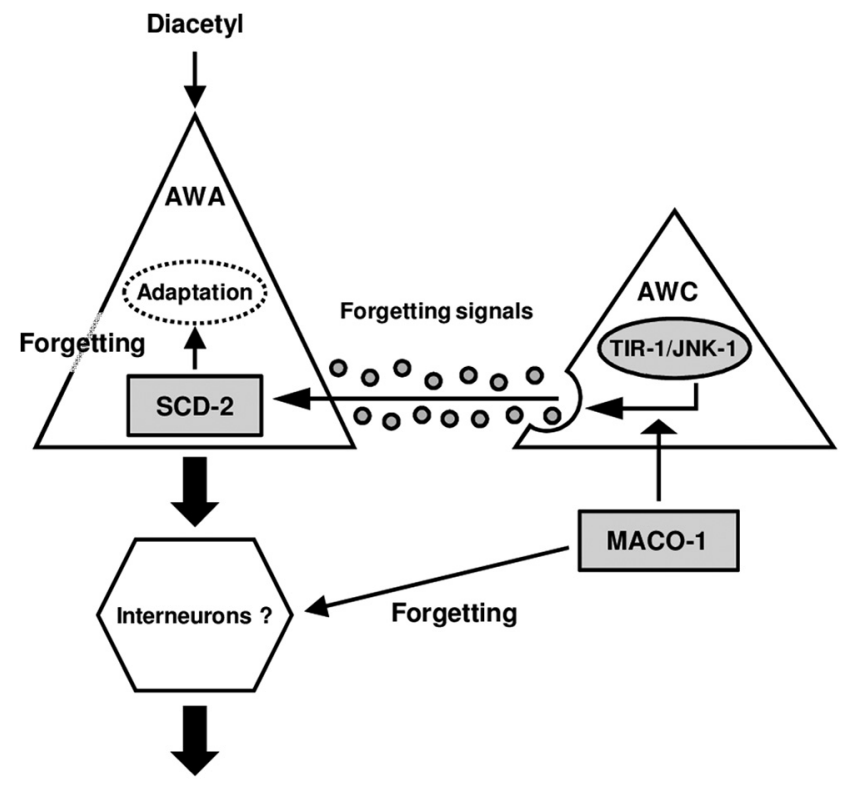

Diacetyl chemoattraction

Figure 7. A model for regulation of forgetting of olfactory adaptation to diacetyl. The genetic epistasis analyses in this study showed that MACO-1 and SCD-2 regulate forgetting of olfactory adaptation to diacetyl downstream of the TIR-1/JNK-1 pathway, and calcium imaging analyses suggested that $\mathrm{MAC}-1$ might be involved in two distinct manners upstream of neural secretion of AWA and downstream of AWA sensation, and that SCD-2 functions for forgetting in AWA sensory neurons.

sensor YC3.60 might affect the $\mathrm{Ca}^{2+}$ response and behavioral phenotype in the maco-1 mutant.

\section{SCD-2 in AWA neurons regulates recovery of calcium} response after olfactory adaptation

The epistasis analyses between SCD-2 and the TIR-1/JNK-1 pathway suggest that SCD-2 regulates forgetting downstream of this pathway. In addition, the rescue experiments of $\mathrm{Ca}^{2+}$ response after the recovery suggested that SCD-2 in AWA neurons is important for the recovery of the sensation. The function of SCD-2 for forgetting in AWA, but not in AWC, is consistent with the result that scd-2 mutant phenotype was not restored by expression of PKC-1 (gf) in AWC neurons, and scd-2 mutants show the defect in forgetting of olfactory adaptation specific to AWA-sensed odorant.

\section{Forgetting of olfactory adaptation may be regulated by multiple signaling pathway}

In this study, we found that MACO- 1 and SCD- 2 regulate forgetting in distinct genetic pathways. As discussed above, MACO-1 might have two functions for the regulation of forgetting; the regulation of the forgetting signals release and the compensation of the unrecovered sensory response, which are upstream of and downstream of AWA sensation, respectively. On the other hand, SCD-2 may function for the regulation of the sensory response in AWA. Since MACO-1 might regulate forgetting in two distinct manners, it appears that MACO-1 and SCD-2 functions upstream of and downstream of the neuronal secretion of AWC, respectively, and that MACO-1 and SCD-2 appear to function in parallel (Fig. 7).

MACO-1 regulates forgetting of olfactory adaptation to both AWA-sensed and AWC-sensed odorants, but SCD-2 regulates forgetting of AWA-sensed adaptation only, suggesting that the regulatory mechanisms of forgetting are distinct in AWA and AWC neurons downstream of the TIR-1/JNK-1 pathway. This study suggests that forgetting of olfactory adaptation is regulated by complicatedly diverged signaling pathway, including the TIR1/JNK-1 pathway, MACO-1, SCD-2, and HEN-1.

In addition, in this study, our genetic epistasis analyses suggest MACO-1 and SCD-2 function downstream of the TIR-1/JNK-1 pathway. However, it is still unclear how these effectors are mechanistically controlled downstream of the TIR-1/JNK-1 pathway. Further study of the signaling pathways mediating these effectors will clarify the precise circuitry mechanism.

\section{Neural secretion may play an important role for forgetting of} olfactory adaptation

Active forgetting may require noncell autonomous regulation by neural secretion. Our previous study suggested that the TIR-1/ JNK-1 pathway accelerated forgetting by facilitating neural secretion from AWC sensory neurons. In Drosophila, activation of the Racl pathway in the mushroom bodies while forgetting olfactory aversive memories requires dopamine release from a small subset of upstream neurons (Shuai et al., 2010; Berry et al., 2012; Cervantes-Sandoval et al., 2016). In this study, we demonstrated that AWC inactivation after conditioning was sufficient to prevent forgetting of olfactory adaptation by histamine-gated chloride channel-induced silencing in AWC neurons. These results imply that signals from AWC neurons during recovery may be important for forgetting. However, although the effect of AWC inactivation during conditioning could not be detected, we cannot exclude the possibility that, in wild-type animals, the function of AWC neurons during conditioning is involved in forgetting.

In our previous study, HEN-1 was predicted to be a secreted protein (Ishihara et al., 2002). Since the expression of HEN-1 has been reported only in AIY and ASE neurons (Ishihara et al., 2002), HEN-1 may not be a forgetting signal secreted from AWC neurons. However, the previous study implied that secreted HEN-1 in the nervous system functioned as an endocrine signal in sensory integration and dauer formation (Reiner et al., 2008; Shinkai et al., 2011), and thus, HEN-1 may function as a secreted signal that regulates forgetting. By identifying the neurons from which HEN-1 is secreted, we will be able to gain a further understanding of the regulatory processes of forgetting.

\section{MACO-1 and SCD-2 function in neuronal regulation in other species}

The previous studies suggested that MACO-1 and SCD-2 were involved in the regulation of several neuronal processes in C. elegans (Reiner et al., 2008; Arellano-Carbajal et al., 2011; Miyara et al., 2011; Shinkai et al., 2011). MACO-1 is a nematode homolog of macoilin, which is a membrane protein conserved across species (Arellano-Carbajal et al., 2011). In mice, macoilin mRNA is highly expressed in specific regions of the brain, such as pyramidal neurons in the hippocampus and granule cells in the dentate gyrus, which are the regions involved in regulation of memory formation (Kuvbachieva et al., 2004). SCD-2 is a nematode homolog of the ALK receptor tyrosine kinase, which is also conserved across species (Hallberg and Palmer, 2013). In mice, ALK shows strong expression throughout the nervous system during embryogenesis, and this expression is maintained at low levels in adult brains (Iwahara et al., 1997; Morris et al., 1997; Vernersson et al., 2006). Because similar expression patterns are observed in rats (Degoutin et al., 2009) and humans (Pulford et al., 1997), it has been suggested that mammalian ALK plays important roles in the development of the nervous system. Furthermore, several studies have suggested the involvement of ALK in regulation of learning in species other than C. elegans. In Drosophila, 
ALK inhibits associative learning in the adult CNS (Gouzi et al., 2011). In mice, ALK redundantly regulates learning of spatial memories with the closely related receptor, leukocyte tyrosine kinase (Weiss et al., 2012). Our study revealed that the functions of MACO-1 and SCD-2 played important roles in noncell autonomous regulation of forgetting in C. elegans, implying that it may be worthwhile to investigate the function of these homologues in forgetting of memories in other species, especially mammals, where the mechanisms of forgetting remain largely unknown.

\section{References}

Altun ZF, Chen B, Wang ZW, Hall DH (2009) High resolution map of Caenorhabditis elegans gap junction proteins. Dev Dyn 238:1936-1950. CrossRef Medline

Arellano-Carbajal F, Briseño-Roa L, Couto A, Cheung BH, Labouesse M, de Bono M (2011) Macoilin, a conserved nervous system-specific ER membrane protein that regulates neuronal excitability. PLoS Genet 7:e1001341. CrossRef Medline

Bargmann CI, Hartwieg E, Horvitz HR (1993) Odorant-selective genes and neurons mediate olfaction in C. elegans. Cell 74:515-527. CrossRef Medline

Berry JA, Cervantes-Sandoval I, Nicholas EP, Davis RL (2012) Dopamine is required for learning and forgetting in Drosophila. Neuron 74:530-542. CrossRef Medline

Bigelow H, Doitsidou M, Sarin S, Hobert O (2009) MAQGene: software to facilitate C. elegans mutant genome sequence analysis. Nat Methods 6:549. CrossRef Medline

Brenner S (1974) The genetics of Caenorhabditis elegans. Genetics 77:71-94. Medline

Brockie PJ, Madsen DM, Zheng Y, Mellem J, Maricq AV (2001) Differential expression of glutamate receptor subunits in the nervous system of Caenorhabditis elegans and their regulation by the homeodomain protein UNC-42. J Neurosci 21:1510-1522. Medline

Cervantes-Sandoval I, Chakraborty M, MacMullen C, Davis RL (2016) Scribble scaffolds a signalosome for active forgetting. Neuron 90:12301242. CrossRef Medline

Chou JH, Bargmann CI, Sengupta P (2001) The Caenorhabditis elegans $o d r-2$ gene encodes a novel Ly-6-related protein required for olfaction. Genetics 157:211-224. Medline

Colbert HA, Bargmann CI (1995) Odorant-specific adaptation pathways generate olfactory plasticity in C. elegans. Neuron 14:803-812. CrossRef Medline

Degoutin J, Brunet-de Carvalho N, Cifuentes-Diaz C, Vigny M (2009) ALK (anaplastic lymphoma kinase) expression in DRG neurons and its involvement in neuron-Schwann cells interaction. Eur J Neurosci 29:275286. CrossRef Medline

Feng Z, Li W, Ward A, Piggott BJ, Larkspur ER, Sternberg PW, Xu XZ (2006) A C. elegans model of nicotine-dependent behavior: regulation by TRPfamily channels. Cell 127:621-633. CrossRef Medline

Gouzi JY, Moressis A, Walker JA, Apostolopoulou AA, Palmer RH, Bernards A, Skoulakis EM (2011) The receptor tyrosine kinase Alk controls neurofibromin functions in Drosophila growth and learning. PLoS Genet 7:e1002281. CrossRef Medline

Hadziselimovic N, Vukojevic V, Peter F, Milnik A, Fastenrath M, Fenyves BG, Hieber P, Demougin P, Vogler C, de Quervain DJ, Papassotiropoulos A, Stetak A (2014) Forgetting is regulated via Musashi-mediated translational control of the Arp2/3 complex. Cell 156:1153-1166. CrossRef Medline

Hallberg B, Palmer RH (2013) Mechanistic insight into ALK receptor tyrosine kinase in human cancer biology. Nat Rev Cancer 13:685-700. CrossRef Medline

Inoue A, Sawatari E, Hisamoto N, Kitazono T, Teramoto T, Fujiwara M, Matsumoto K, Ishihara T (2013) Forgetting in C. elegans is accelerated by neuronal communication via the TIR-1/JNK-1 pathway. Cell Rep 3:808-819. CrossRef Medline

Ishihara T, Iino Y, Mohri A, Mori I, Gengyo-Ando K, Mitani S, Katsura I (2002) HEN-1, a secretory protein with an LDL receptor motif, regulates sensory integration and learning in Caenorhabditis elegans. Cell 109:639649. CrossRef Medline

Iwahara T, Fujimoto J, Wen D, Cupples R, Bucay N, Arakawa T, Mori S, Ratzkin B, Yamamoto T (1997) Molecular characterization of ALK, a receptor tyrosine kinase expressed specifically in the nervous system. Oncogene 14:439-449. CrossRef Medline
Kandel ER, Dudai Y, Mayford MR (2014) The molecular and systems biology of memory. Cell 157:163-186. CrossRef Medline

Kraemer PJ, Golding JM (1997) Adaptive forgetting in animals. Psychon Bull Rev 4:480-491. CrossRef

Krieger M, Herz J (1994) Structures and functions of multiligand lipoprotein receptors: macrophage scavenger receptors and LDL receptor-related protein (LRP). Annu Rev Biochem 63:601-637. CrossRef Medline

Kuvbachieva A, Bestel AM, Tissir F, Maloum I, Guimiot F, Ramoz N, Bourgeois F, Moalic JM, Goffinet AM, Simonneau M (2004) Identification of a novel brain-specific and Reelin-regulated gene that encodes a protein colocalized with synapsin. Eur J Neurosci 20:603-610. CrossRef Medline

L'Etoile ND, Coburn CM, Eastham J, Kistler A, Gallegos G, Bargmann CI (2002) The cyclic GMP-dependent protein kinase EGL-4 regulates olfactory adaptation in C. elegans. Neuron 36:1079-1089. CrossRef Medline

Miyara A, Ohta A, Okochi Y, Tsukada Y, Kuhara A, Mori I (2011) Novel and conserved protein macoilin is required for diverse neuronal functions in Caenorhabditis elegans. PLoS Genet 7:e1001384. CrossRef Medline

Morris SW, Naeve C, Mathew P, James PL, Kirstein MN, Cui X, Witte DP (1997) ALK, the chromosome 2 gene locus altered by the $t(2 ; 5)$ in nonHodgkin's lymphoma, encodes a novel neural receptor tyrosine kinase that is highly related to leukocyte tyrosine kinase (LTK). Oncogene 14: 2175-2188. CrossRef Medline

Nagai T, Yamada S, Tominaga T, Ichikawa M, Miyawaki A (2004) Expanded dynamic range of fluorescent indicators for $\mathrm{Ca}(2+)$ by circularly permuted yellow fluorescent proteins. Proc Natl Acad Sci U S A 101: 10554-10559. CrossRef Medline

Nuttley WM, Atkinson-Leadbeater KP, Van Der Kooy D (2002) Serotonin mediates food-odor associative learning in the nematode Caenorhabditis elegans. Proc Natl Acad Sci U S A 99:12449-12454. CrossRef Medline

Palmitessa A, Hess HA, Bany IA, Kim YM, Koelle MR, Benovic JL (2005) Caenorhabditis elegans arrestin regulates neural $G$ protein signaling and olfactory adaptation and recovery. J Biol Chem 280:24649-24662. CrossRef Medline

Pokala N, Liu Q, Gordus A, Bargmann CI (2014) Inducible and titratable silencing of Caenorhabditis elegans neurons in vivo with histamine-gated chloride channels. Proc Natl Acad Sci U S A 111:2770-2775. CrossRef Medline

Pulford K, Lamant L, Morris SW, Butler LH, Wood KM, Stroud D, Delsol G, Mason DY (1997) Detection of anaplastic lymphoma kinase (ALK) and nucleolar protein nucleophosmin (NPM)-ALK proteins in normal and neoplastic cells with the monoclonal antibody ALK1. Blood 89:13941404. Medline

Reiner DJ, Ailion M, Thomas JH, Meyer BJ (2008) C. elegans anaplastic lymphoma kinase ortholog SCD-2 controls dauer formation by modulating TGF- $\beta$ signaling. Curr Biol 18:1101-1109. CrossRef Medline

Shinkai Y, Yamamoto Y, Fujiwara M, Tabata T, Murayama T, Hirotsu T, Ikeda DD, Tsunozaki M, Iino Y, Bargmann CI, Katsura I, Ishihara T (2011) Behavioral choice between conflicting alternatives is regulated by a receptor guanylyl cyclase, GCY-28, and a receptor tyrosine kinase, SCD-2, in AIA interneurons of Caenorhabditis elegans. J Neurosci 31: 3007-3015. CrossRef Medline

Shuai Y, Lu B, Hu Y, Wang L, Sun K, Zhong Y (2010) Forgetting is regulated through Rac activity in Drosophila. Cell 140:579-589. CrossRef Medline

Sieburth D, Madison JM, Kaplan JM (2007) PKC-1 regulates secretion of neuropeptides. Nat Neurosci 10:49-57. CrossRef Medline

Vernersson E, Khoo NK, Henriksson ML, Roos G, Palmer RH, Hallberg B (2006) Characterization of the expression of the ALK receptor tyrosine kinase in mice. Gene Expr Patterns 6:448-461. CrossRef Medline

Vukojevic V, Gschwind L, Vogler C, Demougin P, de Quervain DJ, Papassotiropoulos A, Stetak A (2012) A role for $\alpha$-adducin (ADD-1) in nematode and human memory. EMBO J 31:1453-1466. CrossRef Medline

Weiss JB, Suyama KL, Lee HH, Scott MP (2001) Jelly belly: a Drosophila LDL receptor repeat-containing signal required for mesoderm migration and differentiation. Cell 107:387-398. CrossRef Medline

Weiss JB, Xue C, Benice T, Xue L, Morris SW, Raber J (2012) Anaplastic lymphoma kinase and leukocyte tyrosine kinase: functions and genetic interactions in learning, memory and adult neurogenesis. Pharmacol Biochem Behav 100:566-574. CrossRef Medline

Zhang C, Yan J, Chen Y, Chen C, Zhang K, Huang X (2014) The olfactory signal transduction for attractive odorants in Caenorhabditis elegans. Biotechnol Adv 32:290-295. CrossRef Medline 